\title{
Study of the $B^{-} \rightarrow J / \psi K^{-} \pi^{+} \pi^{-}$Decay and Measurement of the $B^{-} \rightarrow X(3872) K^{-}$ Branching Fraction.
}

\section{B. Aubert, ${ }^{1}$ R. Barate, ${ }^{1}$ D. Boutigny, ${ }^{1}$ F. Couderc,${ }^{1}$ J.-M. Gaillard, ${ }^{1}$ A. Hicheur,${ }^{1}$ Y. Karyotakis,${ }^{1}$ J. P. Lees,${ }^{1}$}

V. Tisserand, ${ }^{1}$ A. Zghiche, ${ }^{1}$ A. Palano, ${ }^{2}$ A. Pompili, ${ }^{2}$ J. C. Chen,${ }^{3}$ N. D. Qi,${ }^{3}$ G. Rong, ${ }^{3}$ P. Wang, ${ }^{3}$ Y. S. Zhu, ${ }^{3}$

G. Eigen,${ }^{4}$ I. Ofte ${ }^{4}$ B. Stugu, ${ }^{4}$ G. S. Abrams,${ }^{5}$ A. W. Borgland,${ }^{5}$ A. B. Breon,${ }^{5}$ D. N. Brown, ${ }^{5}$ J. Button-Shafer,${ }^{5}$ R. N. Cahn, ${ }^{5}$ E. Charles,${ }^{5}$ C. T. Day,${ }^{5}$ M. S. Gill, ${ }^{5}$ A. V. Gritsan, ${ }^{5}$ Y. Groysman, ${ }^{5}$ R. G. Jacobsen,${ }^{5}$ R. W. Kadel, ${ }^{5}$

J. Kadyk ${ }^{5}$ L. T. Kerth, ${ }^{5}$ Yu. G. Kolomensky, ${ }^{5}$ G. Kukartsev,${ }^{5}$ C. LeClerc, ${ }^{5}$ G. Lynch, ${ }^{5}$ A. M. Merchant, ${ }^{5}$

L. M. Mir, ${ }^{5}$ P. J. Oddone,${ }^{5}$ T. J. Orimoto, ${ }^{5}$ M. Pripstein,${ }^{5}$ N. A. Roe, ${ }^{5}$ M. T. Ronan,${ }^{5}$ V. G. Shelkov,${ }^{5}$

W. A. Wenzel, ${ }^{5}$ K. Ford,${ }^{6}$ T. J. Harrison, ${ }^{6}$ C. M. Hawkes ${ }^{6}$ S. E. Morgan, ${ }^{6}$ A. T. Watson, ${ }^{6}$ M. Fritsch, ${ }^{7}$ K. Goetzen, ${ }^{7}$ T. Held,${ }^{7}$ H. Koch,${ }^{7}$ B. Lewandowski,${ }^{7}$ M. Pelizaeus,${ }^{7}$ M. Steinke, ${ }^{7}$ J. T. Boyd,${ }^{8}$ N. Chevalier,${ }^{8}$ W. N. Cottingham, ${ }^{8}$ M. P. Kelly ${ }^{8}$ T. E. Latham ${ }^{8}$ F. F. Wilson,${ }^{8}$ T. Cuhadar-Donszelmann, ${ }^{9}$ C. Hearty, ${ }^{9}$ N. S. Knecht, ${ }^{9}$ T. S. Mattison, ${ }^{9}$ J. A. McKenna, ${ }^{9}$ D. Thiessen,${ }^{9}$ A. Khan,${ }^{10}$ P. Kyberd,${ }^{10}$ L. Teodorescu, ${ }^{10}$ V. E. Blinov, ${ }^{11}$ A. D. Bukin, ${ }^{11}$

V. P. Druzhinin, ${ }^{11}$ V. B. Golubev, ${ }^{11}$ V. N. Ivanchenko, ${ }^{11}$ E. A. Kravchenko, ${ }^{11}$ A. P. Onuchin, ${ }^{11}$ S. I. Serednyakov, ${ }^{11}$

Yu. I. Skovpen, ${ }^{11}$ E. P. Solodov,${ }^{11}$ A. N. Yushkov,${ }^{11}$ D. Best,${ }^{12}$ M. Bruinsma, ${ }^{12}$ M. Chao, ${ }^{12}$ I. Eschrich,${ }^{12}$

D. Kirkby, ${ }^{12}$ A. J. Lankford, ${ }^{12}$ M. Mandelkern,${ }^{12}$ R. K. Mommsen, ${ }^{12}$ W. Roethel,${ }^{12}$ D. P. Stoker,${ }^{12}$ C. Buchanan, ${ }^{13}$

B. L. Hartfiel, ${ }^{13}$ J. W. Gary, ${ }^{14}$ B. C. Shen, ${ }^{14}$ K. Wang, ${ }^{14}$ D. del Re,${ }^{15}$ H. K. Hadavand, ${ }^{15}$ E. J. Hill, ${ }^{15}$

D. B. MacFarlane, ${ }^{15}$ H. P. Paar, ${ }^{15}$ Sh. Rahatlou,${ }^{15}$ V. Sharma,${ }^{15}$ J. W. Berryhill, ${ }^{16}$ C. Campagnari, ${ }^{16}$ B. Dahmes,${ }^{16}$ S. L. Levy ${ }^{16}$ O. Long, ${ }^{16}$ A. Lu, ${ }^{16}$ M. A. Mazur,${ }^{16}$ J. D. Richman, ${ }^{16}$ W. Verkerke, ${ }^{16}$ T. W. Beck, ${ }^{17}$ A. M. Eisner,${ }^{17}$ C. A. Heusch, ${ }^{17}$ W. S. Lockman, ${ }^{17}$ T. Schalk,${ }^{17}$ R. E. Schmitz, ${ }^{17}$ B. A. Schumm, ${ }^{17}$ A. Seiden, ${ }^{17}$ P. Spradlin, ${ }^{17}$ D. C. Williams, ${ }^{17}$ M. G. Wilson, ${ }^{17}$ J. Albert,${ }^{18}$ E. Chen,${ }^{18}$ G. P. Dubois-Felsmann, ${ }^{18}$ A. Dvoretskii, ${ }^{18}$ D. G. Hitlin,${ }^{18}$

I. Narsky ${ }^{18}$ T. Piatenko, ${ }^{18}$ F. C. Porter,${ }^{18}$ A. Ryd,${ }^{18}$ A. Samuel, ${ }^{18}$ S. Yang, ${ }^{18}$ S. Jayatilleke,${ }^{19}$ G. Mancinelli, ${ }^{19}$ B. T. Meadows, ${ }^{19}$ M. D. Sokoloff, ${ }^{19}$ T. Abe,${ }^{20}$ F. Blanc, ${ }^{20}$ P. Bloom,${ }^{20}$ S. Chen,${ }^{20}$ W. T. Ford,${ }^{20}$ U. Nauenberg, ${ }^{20}$ A. Olivas, ${ }^{20}$ P. Rankin, ${ }^{20}$ J. G. Smith,${ }^{20}$ J. Zhang, ${ }^{20}$ L. Zhang, ${ }^{20}$ A. Chen, ${ }^{21}$ J. L. Harton, ${ }^{21}$ A. Soffer,${ }^{21}$

W. H. Toki ${ }^{21}$ R. J. Wilson, ${ }^{21}$ Q. L. Zeng, ${ }^{21}$ D. Altenburg, ${ }^{22}$ T. Brandt,${ }^{22}$ J. Brose, ${ }^{22}$ T. Colberg, ${ }^{22}$ M. Dickopp,${ }^{22}$

E. Feltresi ${ }^{22}$ A. Hauke, ${ }^{22}$ H. M. Lacker ${ }^{22}$ E. Maly, ${ }^{22}$ R. Müller-Pfefferkorn,${ }^{22}$ R. Nogowski, ${ }^{22}$ S. Otto, ${ }^{22}$

A. Petzold, ${ }^{22}$ J. Schubert, ${ }^{22}$ K. R. Schubert,${ }^{22}$ R. Schwierz, ${ }^{22}$ B. Spaan, ${ }^{22}$ J. E. Sundermann, ${ }^{22}$ D. Bernard, ${ }^{23}$ G. R. Bonneaud, ${ }^{23}$ F. Brochard, ${ }^{23}$ P. Grenier, ${ }^{23}$ S. Schrenk, ${ }^{23}$ Ch. Thiebaux,${ }^{23}$ G. Vasileiadis, ${ }^{23}$ M. Verderi, ${ }^{23}$

D. J. Bard,${ }^{24}$ P. J. Clark, ${ }^{24}$ D. Lavin,${ }^{24}$ F. Muheim,${ }^{24}$ S. Playfer,${ }^{24}$ Y. Xie,${ }^{24}$ M. Andreotti, ${ }^{25}$ V. Azzolini,${ }^{25}$ D. Bettoni, ${ }^{25}$ C. Bozzi,${ }^{25}$ R. Calabrese,${ }^{25}$ G. Cibinetto, ${ }^{25}$ E. Luppi,${ }^{25}$ M. Negrini, ${ }^{25}$ L. Piemontese,${ }^{25}$ A. Sarti, ${ }^{25}$ E. Treadwell, ${ }^{26}$ R. Baldini-Ferroli, ${ }^{27}$ A. Calcaterra,${ }^{27}$ R. de Sangro, ${ }^{27}$ G. Finocchiaro,${ }^{27}$ P. Patteri, ${ }^{27}$ M. Piccolo, ${ }^{27}$ A. Zallo, ${ }^{27}$ A. Buzzo,${ }^{28}$ R. Capra, ${ }^{28}$ R. Contri, ${ }^{28}$ G. Crosetti, ${ }^{28}$ M. Lo Vetere,${ }^{28}$ M. Macri,${ }^{28}$ M. R. Monge,${ }^{28}$ S. Passaggio, ${ }^{28}$ C. Patrignani,${ }^{28}$ E. Robutti, ${ }^{28}$ A. Santroni, ${ }^{28}$ S. Tosi, ${ }^{28}$ S. Bailey, ${ }^{29}$ G. Brandenburg, ${ }^{29}$ M. Morii,${ }^{29}$ E. Won, ${ }^{29}$ R. S. Dubitzky, ${ }^{30}$ U. Langenegger,${ }^{30}$ W. Bhimji, ${ }^{31}$ D. A. Bowerman, ${ }^{31}$ P. D. Dauncey,${ }^{31}$ U. Egede,${ }^{31}$ J. R. Gaillard ${ }^{31}$ G. W. Morton, ${ }^{31}$ J. A. Nash, ${ }^{31}$ G. P. Taylor,${ }^{31}$ G. J. Grenier,${ }^{32}$ U. Mallik, ${ }^{32}$ J. Cochran, ${ }^{33}$ H. B. Crawley, ${ }^{33}$ J. Lamsa, ${ }^{33}$ W. T. Meyer,${ }^{33}$ S. Prell, ${ }^{33}$ E. I. Rosenberg, ${ }^{33}$ J. Yi,${ }^{33}$ M. Davier, ${ }^{34}$ G. Grosdidier,${ }^{34}$ A. Höcker ${ }^{34}$ S. Laplace, ${ }^{34}$ F. Le Diberder,${ }^{34}$ V. Lepeltier, ${ }^{34}$ A. M. Lutz,${ }^{34}$ T. C. Petersen, ${ }^{34}$ S. Plaszczynski,,${ }^{34}$ M. H. Schune,${ }^{34}$ L. Tantot,${ }^{34}$ G. Wormser, ${ }^{34}$ C. H. Cheng, ${ }^{35}$ D. J. Lange,${ }^{35}$ M. C. Simani,,${ }^{35}$ D. M. Wright,${ }^{35}$

A. J. Bevan, ${ }^{36}$ J. P. Coleman, ${ }^{36}$ J. R. Fry ${ }^{36}$ E. Gabathuler ${ }^{36}$ R. Gamet, ${ }^{36}$ R. J. Parry, ${ }^{36}$ D. J. Payne ${ }^{36}$

R. J. Sloane, ${ }^{36}$ C. Touramanis, ${ }^{36}$ J. J. Back, ${ }^{37}$ C. M. Cormack, ${ }^{37}$ P. F. Harrison, ${ }^{37}$, 6 G. B. Mohanty, ${ }^{37}$

C. L. Brown, ${ }^{38}$ G. Cowan, ${ }^{38}$ R. L. Flack, ${ }^{38}$ H. U. Flaecher, ${ }^{38}$ M. G. Green, ${ }^{38}$ C. E. Marker,${ }^{38}$ T. R. McMahon, ${ }^{38}$ S. Ricciardi, ${ }^{38}$ F. Salvatore, ${ }^{38}$ G. Vaitsas, ${ }^{38}$ M. A. Winter,${ }^{38}$ D. Brown, ${ }^{39}$ C. L. Davis, ${ }^{39}$ J. Allison, ${ }^{40}$ N. R. Barlow ${ }^{40}$ R. J. Barlow, ${ }^{40}$ P. A. Hart, ${ }^{40}$ M. C. Hodgkinson, ${ }^{40}$ G. D. Lafferty ${ }^{40}$ A. J. Lyon, ${ }^{40}$ J. C. Williams, ${ }^{40}$ A. Farbin ${ }^{41}$ W. D. Hulsbergen, ${ }^{41}$ A. Jawahery,${ }^{41}$ D. Kovalskyi ${ }^{41}$ C. K. Lae,${ }^{41}$ V. Lillard ${ }^{41}$ D. A. Roberts,${ }^{41}$ G. Blaylock, ${ }^{42}$ C. Dallapiccola ${ }^{42}$ K. T. Flood, ${ }^{42}$ S. S. Hertzbach, ${ }^{42}$ R. Kofler,${ }^{42}$ V. B. Koptchev, ${ }^{42}$ T. B. Moore, ${ }^{42}$ S. Saremi, ${ }^{42}$ 
H. Staengle, ${ }^{42}$ S. Willocq, ${ }^{42}$ R. Cowan,${ }^{43}$ G. Sciolla, ${ }^{43}$ F. Taylor, ${ }^{43}$ R. K. Yamamoto,${ }^{43}$ D. J. J. Mangeol, ${ }^{44}$ P. M. Patel ${ }^{44}$ S. H. Robertson, ${ }^{44}$ A. Lazzaro, ${ }^{45}$ F. Palombo, ${ }^{45}$ J. M. Bauer ${ }^{46}$ L. Cremaldi, ${ }^{46}$ V. Eschenburg, ${ }^{46}$ R. Godang, ${ }^{46}$ R. Kroeger, ${ }^{46}$ J. Reidy, ${ }^{46}$ D. A. Sanders, ${ }^{46}$ D. J. Summers ${ }^{46}$ H. W. Zhao, ${ }^{46}$ S. Brunet ${ }^{47}$ D. Côté, ${ }^{47}$ P. Taras,${ }^{47}$ H. Nicholson, ${ }^{48}$ N. Cavallo, ${ }^{49}$ F. Fabozzi, ${ }^{49}$, ${ }^{4}$ C. Gatto, ${ }^{49}$ L. Lista,${ }^{49}$ D. Monorchio, ${ }^{49}$ P. Paolucci ${ }^{49}$ D. Piccolo, ${ }^{49}$ C. Sciacca, ${ }^{49}$ M. Baak,${ }^{50}$ H. Bulten,${ }^{50}$ G. Raven,${ }^{50}$ L. Wilden,${ }^{50}$ C. P. Jessop, ${ }^{51}$ J. M. LoSecco, ${ }^{51}$

T. A. Gabriel ${ }^{52}$ T. Allmendinger, ${ }^{53}$ B. Brau, ${ }^{53}$ K. K. Gan, ${ }^{53}$ K. Honscheid,${ }^{53}$ D. Hufnagel,${ }^{53}$ H. Kagan, ${ }^{53}$ R. Kass, ${ }^{53}$ T. Pulliam, ${ }^{53}$ A. M. Rahimi,${ }^{53}$ R. Ter-Antonyan, ${ }^{53}$ Q. K. Wong, ${ }^{53}$ J. Brau,${ }^{54}$ R. Frey, ${ }^{54}$ O. Igonkina, ${ }^{54}$ C. T. Potter ${ }^{54}$ N. B. Sinev ${ }^{54}$ D. Strom,${ }^{54}$ E. Torrence,${ }^{54}$ F. Colecchia,${ }^{55}$ A. Dorigo,${ }^{55}$ F. Galeazzi,${ }^{55}$ M. Margoni,${ }^{55}$ M. Morandin, ${ }^{55}$ M. Posocco, ${ }^{55}$ M. Rotondo,${ }^{55}$ F. Simonetto, ${ }^{55}$ R. Stroili, ${ }^{55}$ G. Tiozzo,${ }^{55}$ C. Voci,${ }^{55}$ M. Benayoun, ${ }^{56}$

H. Briand, ${ }^{56}$ J. Chauveau, ${ }^{56}$ P. David,${ }^{56}$ Ch. de la Vaissière, ${ }^{56}$ L. Del Buono, ${ }^{56}$ O. Hamon, ${ }^{56}$ M. J. J. John, ${ }^{56}$ Ph. Leruste ${ }^{56}$ J. Ocariz ${ }^{56}$ M. Pivk ${ }^{56}$ L. Roos ${ }^{56}$ S. T'Jampens, ${ }^{56}$ G. Therin, ${ }^{56}$ P. F. Manfredi, ${ }^{57}$ V. Re,${ }^{57}$ P. K. Behera, ${ }^{58}$ L. Gladney, ${ }^{58}$ Q. H. Guo, ${ }^{58}$ J. Panetta, ${ }^{58}$ F. Anulli, ${ }^{27,}{ }^{59}$ M. Biasini, ${ }^{59}$ I. M. Peruzzi, ${ }^{27,}{ }^{59}$ M. Pioppi,${ }^{59}$ C. Angelini, ${ }^{60}$ G. Batignani, ${ }^{60}$ S. Bettarini, ${ }^{60}$ M. Bondioli,${ }^{60}$ F. Bucci,${ }^{60}$ G. Calderini,,${ }^{60}$ M. Carpinelli, ${ }^{60}$ V. Del Gamba, ${ }^{60}$ F. Forti, ${ }^{60}$ M. A. Giorgi,${ }^{60}$ A. Lusiani, ${ }^{60}$ G. Marchiori, ${ }^{60}$ F. Martinez-Vidal, ${ }^{60 \text {, 团 }}$ M. Morganti, ${ }^{60}$ N. Neri, ${ }^{60}$ E. Paoloni, ${ }^{60}$ M. Rama,${ }^{60}$ G. Rizzo, ${ }^{60}$ F. Sandrelli, ${ }^{60}$ J. Walsh, ${ }^{60}$ M. Haire, ${ }^{61}$ D. Judd, ${ }^{61}$ K. Paick, ${ }^{61}$ D. E. Wagoner, ${ }^{61}$ N. Danielson, ${ }^{62}$ P. Elmer,${ }^{62}$ Y. P. Lau ${ }^{62}$ C. Lu ${ }^{62}$ V. Miftakov, ${ }^{62}$ J. Olsen, ${ }^{62}$ A. J. S. Smith, ${ }^{62}$ A. V. Telnov, ${ }^{62}$ F. Bellini, ${ }^{63}$ G. Cavoto, ${ }^{62}{ }^{63}$ R. Faccini,${ }^{63}$ F. Ferrarotto,${ }^{63}$ F. Ferroni,${ }^{63}$ M. Gaspero, ${ }^{63}$ L. Li Gioi, ${ }^{63}$ M. A. Mazzoni,${ }^{63}$ S. Morganti, ${ }^{63}$ M. Pierini, ${ }^{63}$ G. Piredda,${ }^{63}$ F. Safai Tehrani,${ }^{63}$ C. Voena ${ }^{63}$ S. Christ,${ }^{64}$ G. Wagner, ${ }^{64}$ R. Waldi,${ }^{64}$ T. Adye,${ }^{65}$ N. De Groot,${ }^{65}$ B. Franek, ${ }^{65}$ N. I. Geddes,${ }^{65}$ G. P. Gopal, ${ }^{65}$ E. O. Olaiya, ${ }^{65}$ R. Aleksan, ${ }^{66}$ S. Emery, ${ }^{66}$ A. Gaidot,${ }^{66}$ S. F. Ganzhur,${ }^{66}$ P.-F. Giraud,${ }^{66}$ G. Hamel de Monchenault, ${ }^{66}$ W. Kozanecki, ${ }^{66}$ M. Langer,${ }^{66}$ M. Legendre ${ }^{66}$ G. W. London, ${ }^{66}$ B. Mayer,${ }^{66}$ G. Schott, ${ }^{66}$ G. Vasseur, ${ }^{66}$ Ch. Yèche ${ }^{66}$ M. Zito ${ }^{66}$ M. V. Purohit, ${ }^{67}$ A. W. Weidemann, ${ }^{67}$ F. X. Yumiceva, ${ }^{67}$ D. Aston, ${ }^{68}$ R. Bartoldus ${ }^{68}$ N. Berger, ${ }^{68}$ A. M. Boyarski, ${ }^{68}$ O. L. Buchmueller, ${ }^{68}$ M. R. Convery, ${ }^{68}$ M. Cristinziani, ${ }^{68}$ G. De Nardo, ${ }^{68}$ D. Dong, ${ }^{68}$ J. Dorfan, ${ }^{68}$ D. Dujmic, ${ }^{68}$ W. Dunwoodie, ${ }^{68}$ E. E. Elsen, ${ }^{68}$ S. Fan, ${ }^{68}$ R. C. Field, ${ }^{68}$ T. Glanzman, ${ }^{68}$ S. J. Gowdy, ${ }^{68}$ T. Hadig, ${ }^{68}$ V. Halyo, ${ }^{68}$ C. Hast,${ }^{68}$ T. Hryn'ova, ${ }^{68}$ W. R. Innes,${ }^{68}$ M. H. Kelsey ${ }^{68}$ P. Kim, ${ }^{68}$ M. L. Kocian, ${ }^{68}$ D. W. G. S. Leith,${ }^{68}$ J. Libby, ${ }^{68}$ S. Luitz ${ }^{68}$ V. Luth,${ }^{68}$ H. L. Lynch, ${ }^{68}$ H. Marsiske, ${ }^{68}$ R. Messner ${ }^{68}$ D. R. Muller, ${ }^{68}$ C. P. O'Grady,${ }^{68}$ V. E. Ozcan, ${ }^{68}$ A. Perazzo,${ }^{68}$ M. Perl, ${ }^{68}$ S. Petrak,${ }^{68}$ B. N. Ratcliff, ${ }^{68}$ A. Roodman, ${ }^{68}$ A. A. Salnikov, ${ }^{68}$ R. H. Schindler, ${ }^{68}$ J. Schwiening, ${ }^{68}$ G. Simi, ${ }^{68}$ A. Snyder, ${ }^{68}$ A. Soha, ${ }^{68}$ J. Stelzer ${ }^{68}$ D. Su${ }^{68}$ M. K. Sullivan, ${ }^{68}$ J. Va'vra, ${ }^{68}$ S. R. Wagner, ${ }^{68}$ M. Weaver, ${ }^{68}$ A. J. R. Weinstein, ${ }^{68}$ W. J. Wisniewski, ${ }^{68}$ M. Wittgen, ${ }^{68}$ D. H. Wright,${ }^{68}$ A. K. Yarritu, ${ }^{68}$ C. C. Young, ${ }^{68}$ P. R. Burchat,${ }^{69}$

A. J. Edwards, ${ }^{69}$ T. I. Meyer ${ }^{69}$ B. A. Petersen, ${ }^{69}$ C. Roat, ${ }^{69}$ S. Ahmed,${ }^{70}$ M. S. Alam,${ }^{70}$ J. A. Ernst, ${ }^{70}$ M. A. Saeed, ${ }^{70}$ M. Saleem, ${ }^{70}$ F. R. Wappler ${ }^{70}$ W. Bugg, ${ }^{71}$ M. Krishnamurthy,${ }^{71}$ S. M. Spanier, ${ }^{71}$ R. Eckmann,${ }^{72}$ H. Kim, ${ }^{72}$ J. L. Ritchie, ${ }^{72}$ A. Satpathy,${ }^{72}$ R. F. Schwitters,${ }^{72}$ J. M. Izen, ${ }^{73}$ I. Kitayama, ${ }^{73}$ X. C. Lou, ${ }^{73}$ S. Ye ${ }^{73}$ F. Bianchi, ${ }^{74}$ M. Bona,${ }^{74}$ F. Gallo,${ }^{74}$ D. Gamba,${ }^{74}$ C. Borean,${ }^{75}$ L. Bosisio,${ }^{75}$ C. Cartaro,${ }^{75}$ F. Cossutti, ${ }^{75}$ G. Della Ricca ${ }^{75}$ S. Dittongo,${ }^{75}$ S. Grancagnolo, ${ }^{75}$ L. Lanceri, ${ }^{75}$ P. Poropat $,{ }^{75}, \S$ L. Vitale,${ }^{75}$ G. Vuagnin, ${ }^{75}$ R. S. Panvini, ${ }^{76}$ Sw. Banerjee, ${ }^{77}$ C. M. Brown, ${ }^{77}$ D. Fortin, ${ }^{77}$ P. D. Jackson, ${ }^{77}$ R. Kowalewski, ${ }^{77}$ J. M. Roney, ${ }^{77}$ H. R. Band, ${ }^{78}$ S. Dasu, ${ }^{78}$ M. Datta, ${ }^{78}$ A. M. Eichenbaum,${ }^{78}$ M. Graham,${ }^{78}$ J. J. Hollar,${ }^{78}$ J. R. Johnson, ${ }^{78}$ P. E. Kutter ${ }^{78}$ H. Li ${ }^{78}$ R. Liu, ${ }^{78}$ F. Di Lodovico, ${ }^{78}$ A. Mihalyi, ${ }^{78}$ A. K. Mohapatra, ${ }^{78}$ Y. Pan, ${ }^{78}$ R. Prepost,${ }^{78}$ A. E. Rubin, ${ }^{78}$ S. J. Sekula, ${ }^{78}$ P. Tan, ${ }^{78}$ J. H. von Wimmersperg-Toeller, ${ }^{78}$ J. Wu, ${ }^{78}$ S. L. Wu, ${ }^{78}$ Z. Yu, ${ }^{78}$ and H. Neal ${ }^{79}$

(The BABAR Collaboration)

\author{
${ }^{1}$ Laboratoire de Physique des Particules, F-74941 Annecy-le-Vieux, France \\ ${ }^{2}$ Università di Bari, Dipartimento di Fisica and INFN, I-70126 Bari, Italy \\ ${ }^{3}$ Institute of High Energy Physics, Beijing 100039, China \\ ${ }^{4}$ University of Bergen, Inst. of Physics, N-5007 Bergen, Norway \\ ${ }^{5}$ Lawrence Berkeley National Laboratory and University of California, Berkeley, CA 94720, USA \\ ${ }^{6}$ University of Birmingham, Birmingham, B15 2TT, United Kingdom \\ ${ }^{7}$ Ruhr Universität Bochum, Institut für Experimentalphysik 1, D-44780 Bochum, Germany \\ ${ }^{8}$ University of Bristol, Bristol BS8 1TL, United Kingdom \\ ${ }^{9}$ University of British Columbia, Vancouver, BC, Canada V6T $1 Z 1$ \\ ${ }^{10}$ Brunel University, Uxbridge, Middlesex UB8 3PH, United Kingdom \\ ${ }^{11}$ Budker Institute of Nuclear Physics, Novosibirsk 630090, Russia \\ ${ }^{12}$ University of California at Irvine, Irvine, CA 92697, USA \\ ${ }^{13}$ University of California at Los Angeles, Los Angeles, CA 90024, USA \\ ${ }^{14}$ University of California at Riverside, Riverside, CA 92521, USA
}


${ }^{15}$ University of California at San Diego, La Jolla, CA 92093, USA

${ }^{16}$ University of California at Santa Barbara, Santa Barbara, CA 93106, USA

${ }^{17}$ University of California at Santa Cruz, Institute for Particle Physics, Santa Cruz, CA 95064, USA

${ }^{18}$ California Institute of Technology, Pasadena, CA 91125, USA

${ }^{19}$ University of Cincinnati, Cincinnati, $\mathrm{OH}$ 45221, USA

${ }^{20}$ University of Colorado, Boulder, CO 80309, USA

${ }^{21}$ Colorado State University, Fort Collins, CO 80523, USA

${ }^{22}$ Technische Universität Dresden, Institut für Kern- und Teilchenphysik, D-01062 Dresden, Germany

${ }^{23}$ Ecole Polytechnique, LLR, F-91128 Palaiseau, France

${ }^{24}$ University of Edinburgh, Edinburgh EH9 3JZ, United Kingdom

${ }^{25}$ Università di Ferrara, Dipartimento di Fisica and INFN, I-44100 Ferrara, Italy

${ }^{26}$ Florida A\&M University, Tallahassee, FL 32307, USA

${ }^{27}$ Laboratori Nazionali di Frascati dell'INFN, I-00044 Frascati, Italy

${ }^{28}$ Università di Genova, Dipartimento di Fisica and INFN, I-16146 Genova, Italy

${ }^{29}$ Harvard University, Cambridge, MA 02138, USA

${ }^{30}$ Universität Heidelberg, Physikalisches Institut, Philosophenweg 12, D-69120 Heidelberg, Germany

${ }^{31}$ Imperial College London, London, SW7 2AZ, United Kingdom

${ }^{32}$ University of Iowa, Iowa City, IA 52242, USA

${ }^{33}$ Iowa State University, Ames, IA 50011-3160, USA

${ }^{34}$ Laboratoire de l'Accélérateur Linéaire, F-91898 Orsay, France

${ }^{35}$ Lawrence Livermore National Laboratory, Livermore, CA 94550, USA

${ }^{36}$ University of Liverpool, Liverpool L69 72E, United Kingdom

${ }^{37}$ Queen Mary, University of London, E1 4NS, United Kingdom

${ }^{38}$ University of London, Royal Holloway and Bedford New College, Egham, Surrey TW20 OEX, United Kingdom

${ }^{39}$ University of Louisville, Louisville, KY 40292, USA

${ }^{40}$ University of Manchester, Manchester M13 9PL, United Kingdom

${ }^{41}$ University of Maryland, College Park, MD 20742, USA

${ }^{42}$ University of Massachusetts, Amherst, MA 01003, USA

${ }^{43}$ Massachusetts Institute of Technology, Laboratory for Nuclear Science, Cambridge, MA 02139, USA

${ }^{44}$ McGill University, Montréal, QC, Canada H3A $2 T 8$

${ }^{45}$ Università di Milano, Dipartimento di Fisica and INFN, I-20133 Milano, Italy

${ }^{46}$ University of Mississippi, University, MS 38677, USA

${ }^{47}$ Université de Montréal, Laboratoire René J. A. Lévesque, Montréal, QC, Canada H3C 3J7

${ }^{48}$ Mount Holyoke College, South Hadley, MA 01075, USA

${ }^{49}$ Università di Napoli Federico II, Dipartimento di Scienze Fisiche and INFN, I-80126, Napoli, Italy

${ }^{50}$ NIKHEF, National Institute for Nuclear Physics and High Energy Physics, NL-1009 DB Amsterdam, The Netherlands

${ }^{51}$ University of Notre Dame, Notre Dame, IN 46556, USA

${ }^{52}$ Oak Ridge National Laboratory, Oak Ridge, TN 37831, USA

${ }^{53}$ Ohio State University, Columbus, OH 43210, USA

${ }^{54}$ University of Oregon, Eugene, OR 97403, USA

${ }^{55}$ Università di Padova, Dipartimento di Fisica and INFN, I-35131 Padova, Italy

${ }^{56}$ Universités Paris VI et VII, Lab de Physique Nucléaire H. E., F-75252 Paris, France

${ }^{57}$ Università di Pavia, Dipartimento di Elettronica and INFN, I-27100 Pavia, Italy

${ }^{58}$ University of Pennsylvania, Philadelphia, PA 19104, USA

${ }^{59}$ Università di Perugia, Dipartimento di Fisica and INFN, I-06100 Perugia, Italy

${ }^{60}$ Università di Pisa, Dipartimento di Fisica, Scuola Normale Superiore and INFN, I-56127 Pisa, Italy

${ }^{61}$ Prairie View A\&M University, Prairie View, TX 77446, USA

${ }^{62}$ Princeton University, Princeton, NJ 08544, USA

${ }^{63}$ Università di Roma La Sapienza, Dipartimento di Fisica and INFN, I-00185 Roma, Italy

${ }^{64}$ Universität Rostock, D-18051 Rostock, Germany

${ }^{65}$ Rutherford Appleton Laboratory, Chilton, Didcot, Oxon, OX11 OQX, United Kingdom

${ }^{66}$ DSM/Dapnia, CEA/Saclay, F-91191 Gif-sur-Yvette, France

${ }^{67}$ University of South Carolina, Columbia, SC 29208, USA

${ }^{68}$ Stanford Linear Accelerator Center, Stanford, CA 94309, USA

${ }^{69}$ Stanford University, Stanford, CA 94305-4060, USA

${ }^{70}$ State Univ. of New York, Albany, NY 12222, USA

${ }^{71}$ University of Tennessee, Knoxville, TN 37996, USA

${ }^{72}$ University of Texas at Austin, Austin, TX 78712, USA

${ }^{73}$ University of Texas at Dallas, Richardson, TX 75083, USA

${ }^{74}$ Università di Torino, Dipartimento di Fisica Sperimentale and INFN, I-10125 Torino, Italy

${ }^{75}$ Università di Trieste, Dipartimento di Fisica and INFN, I-34127 Trieste, Italy

${ }^{76}$ Vanderbilt University, Nashville, TN 37235, USA

${ }^{77}$ University of Victoria, Victoria, BC, Canada V8W 3P6

${ }^{78}$ University of Wisconsin, Madison, WI 53706, USA 
${ }^{79}$ Yale University, New Haven, CT 06511, USA

(Dated: June 7, 2004)

\begin{abstract}
We study the decay $B^{-} \rightarrow J / \psi K^{-} \pi^{+} \pi^{-}$using 117 million $B \bar{B}$ events collected at the $Y(4 S)$ resonance with the BaBar detector at the PEP-II $e^{+} e^{-}$asymmetric-energy storage ring. We measure the branching fractions $\mathcal{B}\left(B^{-} \rightarrow J / \psi K^{-} \pi^{+} \pi^{-}\right)=(116 \pm 7$ (stat. $) \pm 9($ syst. $\left.)\right) \times 10^{-5}$ and $\mathcal{B}\left(B^{-} \rightarrow X(3872) K^{-}\right) \times \mathcal{B}\left(X(3872) \rightarrow \mathrm{J} / \psi \pi^{+} \pi^{-}\right)=(1.28 \pm 0.41) \times 10^{-5}$ and find the mass of the $X(3872)$ to be $3873.4 \pm 1.4 \mathrm{MeV} / \mathrm{c}^{2}$. We search for the $h_{c}$ narrow state in the decay $B^{-} \rightarrow h_{c} K^{-}$, $h_{c} \rightarrow J / \psi \pi^{+} \pi^{-}$and for the decay $B^{-} \rightarrow J / \psi D^{0} \pi^{-}$, with $D^{0} \rightarrow K^{-} \pi^{+}$. We set the $90 \%$ C.L. limits $\mathcal{B}\left(B^{-} \rightarrow h_{c} K^{-}\right) \times \mathcal{B}\left(h_{c} \rightarrow J / \psi \pi^{+} \pi^{-}\right)<3.4 \times 10^{-6}$ and $\mathcal{B}\left(B^{-} \rightarrow J / \psi D^{0} \pi^{-}\right)<5.2 \times 10^{-5}$.
\end{abstract}

PACS numbers: 13.25.Hw, 12.15.Hh, 11.30.Er

The study of $B$ decays to final states containing charmonium and strange mesons is especially suited to the search for new charmonium states and for intrinsic charm. In particular, the decay $B^{-} \rightarrow J / \psi K^{-} \pi^{+} \pi^{-}[1]$ can occur via the production of charmonium states decaying into $J / \psi \pi^{+} \pi^{-}$or possibly via $B^{-} \rightarrow J / \psi D^{0} \pi^{-}$, with $D^{0} \rightarrow K^{-} \pi^{+}$. Recently the Belle [2] and CDF [3] collaborations have observed a new state, the $X(3872)$, decaying into $J / \psi \pi^{+} \pi^{-}$. This state is a charmonium candidate, the $1^{3} D_{2}$ or $1^{3} D_{3}[4]$, with $J^{P C}=2^{--}$or $J^{P C}=3^{--}$, or even possibly a molecule of charmed $D$ and $D^{*}$ mesons $[5]$. In this Letter, using 117 million $\Upsilon(4 S)$ decays into $B \bar{B}$ pairs, we confirm the observation of the $X(3872)$ and search for the unconfirmed charmonium $1 P_{1}$ state $h_{c}(3526)$ [6]. In addition, we study final states involving $D$ mesons to test models developed to explain the excess of low momentum $J / \psi$ mesons in inclusive $B$ decays [7].

The data were collected at the PEP-II asymmetricenergy $e^{+} e^{-}$B-factory with the BABAR detector, which is fully described elsewhere [9]. The detector includes a silicon vertex tracker and a drift chamber in a 1.5$\mathrm{T}$ solenoidal magnetic field, which detect charged particles and measure their momentum and energy loss. Photons, electrons, and neutral hadrons are detected in a $\mathrm{CsI}(\mathrm{Tl})$-crystal electromagnetic calorimeter. A ringimaging Cherenkov detector is used for particle identification. Penetrating muons and neutral hadrons are identified by resistive-plate chambers in the steel of the flux return. We use a Monte Carlo simulation of the BABAR detector based on GEANT4 [10] to validate the analysis procedure and to estimate efficiency corrections.

The event reconstruction and selection follow closely those described in an earlier paper 11]. The present analysis has been optimized to maximize the sensitivity to $B^{-} \rightarrow J / \psi K^{-} \pi^{+} \pi^{-}$decays. We reconstruct $J / \psi \rightarrow e^{+} e^{-}$candidates from pairs of tracks selected with criteria that are $98 \%$ (7\%) efficient for electrons (pions). To account for energy losses, we combine the electron pairs with bremsstrahlung-photon candidates and use an asymmetric mass window, $2.95<m_{e e(\gamma)}<3.14 \mathrm{GeV} / c^{2}$. We reconstruct $J / \psi \rightarrow \mu^{+} \mu^{-}$candidates from pairs of tracks selected with criteria that are $77 \%$ (8\%) efficient for muons (pions), satisfying $3.06<m_{\mu \mu}<3.14 \mathrm{GeV} / c^{2}$.
The nominal $J / \psi$ mass 12 is imposed as a constraint on $J / \psi$ candidates, thereby improving the resolution on the $B$ mass and on any charmonium states in its decay. Kaons are identified using criteria that have an efficiency of $97 \%$, with a $15 \%$ pion-misidentification rate. $B$-meson candidates are formed by combining a $J / \psi$ candidate with a kaon candidate and two additional oppositely charged tracks. To suppress further the background from light-quark production, which is characterized by back-to-back jets, the angle $\theta_{T}$ between the thrust axes of the reconstructed $B$ candidate and the rest of the event in the center-of-mass system is required to satisfy $\left|\cos \theta_{T}\right|<0.8(0.9)$ for $J / \psi \rightarrow e^{+} e^{-}\left(J / \psi \rightarrow \mu^{+} \mu^{-}\right)$candidates.

Signal and combinatorial background are discriminated using two kinematic variables: the beam-energysubstituted mass, $m_{\mathrm{ES}} \equiv \sqrt{(\sqrt{s} / 2)^{2}-p_{B}^{*}{ }^{2}}$, and the difference of the $B$ candidate's measured energy from the beam energy, $\Delta E \equiv E_{B}^{*}-(\sqrt{s} / 2)$. Here $E_{B}^{*}\left(p_{B}^{*}\right)$ is the energy (momentum) of the $B$ candidate in the centerof-mass frame and $\sqrt{s}$ is the total center-of-mass energy. The signal region is defined to be $|\Delta E|<3 \sigma$, where the resolution $\sigma$, determined with data, is $12 \mathrm{MeV}$. A binned likelihood fit to the $m_{\mathrm{ES}}$ distribution (Fig. 1(a)) is used to separate the signal, taken as a Gaussian distribution with a fitted width of about $2.5 \mathrm{MeV} / c^{2}$, plus a small tail to account for energy losses [14], from the combinatorial background distributed as an ARGUS threshold function [15]. We have checked with Monte Carlo simulation that there is no significant background from $B$ decays that has the same $m_{\mathrm{ES}}$ distribution as the signal.

To reduce systematic uncertainties, we measure

$$
\begin{aligned}
R & =\frac{\mathcal{B}\left(B^{-} \rightarrow J / \psi K^{-} \pi^{+} \pi^{-}\right)}{\mathcal{B}\left(B^{-} \rightarrow \psi(2 S) K^{-}\right)} \\
& =\frac{N_{\text {events }}}{N_{\psi(2 S)}} \frac{\epsilon_{\psi(2 S)}}{\epsilon} \times \mathcal{B}\left(\psi(2 S) \rightarrow J / \psi \pi^{+} \pi^{-}\right),
\end{aligned}
$$

where $N_{\text {events }}=2540 \pm 72$ is the number of $B^{-} \rightarrow J / \psi K^{-} \pi^{+} \pi^{-}$signal events extracted from the fit to the $m_{\mathrm{ES}}$ distribution. The number of $\psi(2 S)$ events, $N_{\psi(2 S)}=556 \pm 30$, is obtained by fitting $m_{J / \psi \pi \pi}$ distribution, after subratcting combinatorial background, with two Gaussian distributions representing the $\psi(2 S)$ 


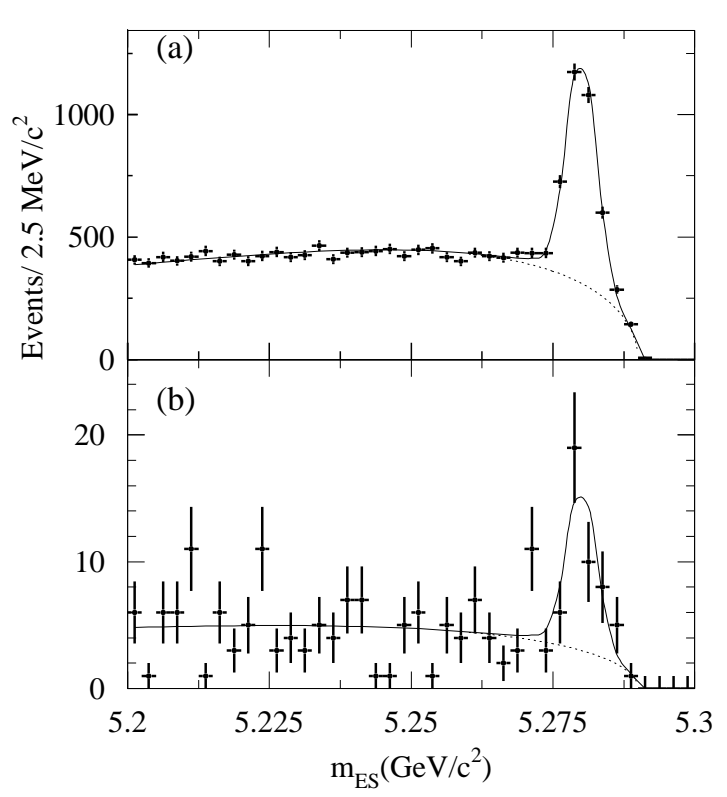

FIG. 1: Distribution of $m_{\mathrm{ES}}$ for (a) $B^{-} \rightarrow J / \psi K^{-} \pi^{+} \pi^{-}$ candidates, and (b) events in the $X(3872)$ region, $3862<$ $m_{J / \psi \pi \pi}<3882 \mathrm{MeV} / c^{2}$. The solid curves represent the binned likelihood fits described in the text; the combinatorial components are indicated by the dashed curves.

signal and a flat distribution representing the remaining background. (Fig. 2(c) shows the corresponding unsubtracted distribution). Throughout this Letter the distributions after combinatorial-background subtraction are obtained by fitting the $m_{\mathrm{ES}}$ distribution of the events within each bin of the variable of interest $\left(m_{J / \psi \pi \pi}\right.$ in this case). The binned $\chi^{2}$ fit gives a resolution on $m_{J / \psi \pi \pi}$ of $3.1 \pm 0.2 \mathrm{MeV} / c^{2}$ for the core Gaussian containing $70 \%$ of the events and $12 \pm 3 \mathrm{MeV} / c^{2}$ for the broader Gaussian. The total $B^{-} \rightarrow J / \psi K^{-} \pi^{+} \pi^{-}$and the $\psi(2 S)$ selection efficiencies, $\epsilon$ and $\epsilon_{\psi(2 S)}$, are extracted from Monte Carlo simulation: we obtain $\epsilon_{\psi(2 S)} / \epsilon=1.17 \pm 0.03$. We use $\mathcal{B}\left(\psi(2 S) \rightarrow J / \psi \pi^{+} \pi^{-}\right)=(31.8 \pm 1.0) \%$ [12].

We estimate the systematic error due to the choice of the signal $m_{\mathrm{ES}}$ shape function by replacing it with a simple Gaussian. We estimate the uncertainty on the fit to the $m_{J / \psi \pi \pi}$ distribution by using the signal resolution function as measured on Monte Carlo and by varying the background shape. Including all these errors, we measure $R=1.70 \pm 0.10$ (stat.) \pm 0.09 (syst.) which, combined with $\mathcal{B}\left(B^{-} \rightarrow \psi(2 S) K^{-}\right)=(6.8 \pm 0.4) \times 10^{-4}[12$, yields

$$
\begin{aligned}
\mathcal{B}\left(B^{-} \rightarrow J / \psi K^{-} \pi^{+} \pi^{-}\right) & = \\
(116 & \pm 7(\text { stat. }) \pm 9(\text { syst. })) \times 10^{-5} .
\end{aligned}
$$

To investigate the possible presence of narrow charmonium states decaying to $J / \psi \pi^{-} \pi^{+}$, we have studied the distribution in $m_{J / \psi \pi \pi}$ (Fig. 2(a)). We observe an

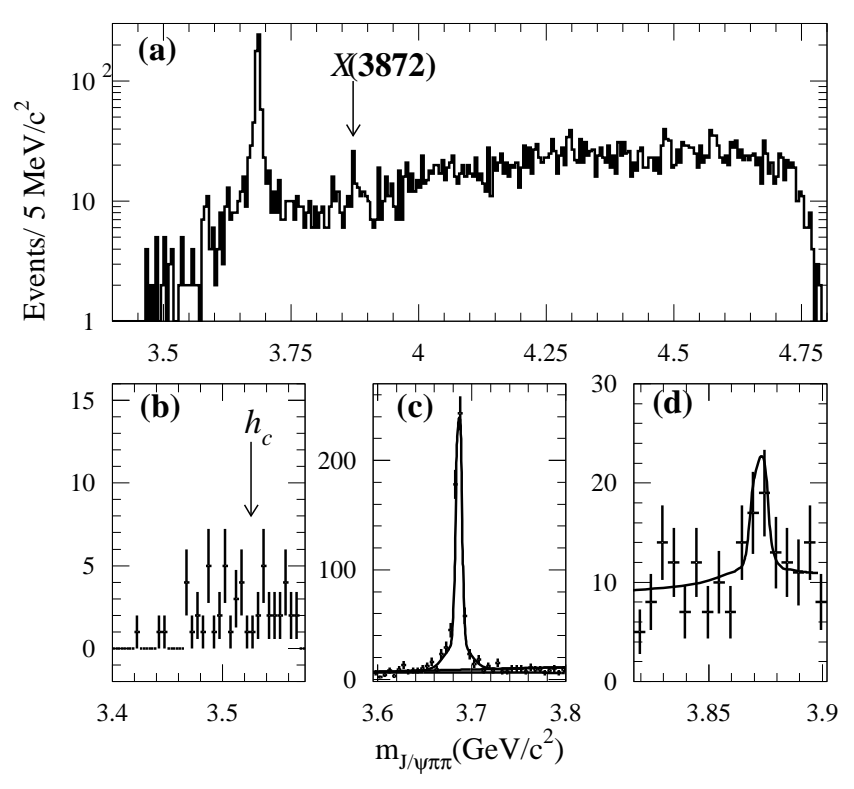

FIG. 2: Distribution of $m_{J / \psi \pi \pi}$ (a) in the entire range, (b) in the $h_{c}$ region, (c) at the $\psi(2 S)$, and (d) in the region of the $X(3872)$ with the projection of the unbinned likelihood fit superimposed. The requirement $m_{\mathrm{ES}}>5.27 \mathrm{GeV} / c^{2}$ is applied.

excess in the region of the $X(3872)$ (Fig. 2(d)), but do not find any excess in the $h_{c}$ region (Fig. 2(b)). The mass of the $X(3872)$ state is extracted from an unbinned maximum likelihood fit to the two-dimensional distribution in $m_{\mathrm{ES}}$ and $m_{J / \psi \pi \pi}$. The probability density function (PDF) is taken to be the sum of four terms. The first three describe $B^{-} \rightarrow J / \psi K^{-} \pi^{+} \pi^{-}$decays that peak when $m_{\mathrm{ES}}$ is the mass of the $B$-meson. The PDF of these three terms contains a Gaussian function in $m_{\mathrm{ES}}$ times a function of $m_{J / \psi \pi \pi}$ that describes: 1) non-resonant events, distributed as a first order polynomial; 2) $\psi(2 S)$ candidates, distributed as a doubleGaussian resolution function around a mean value that is allowed to float; and 3) $X(3872)$ candidates, with the same resolution function as the $\psi(2 S)$ but with a mass that floats relative to the $\psi(2 S)$ mass. The measurement of mass difference allows us to neglect systematic errors on the absolute mass scale. The fourth term of the PDF describes the combinatorial background, distributed as an ARGUS threshold function in $m_{\mathrm{ES}}$ and as a first order polynomial in $m_{J / \psi \pi \pi}$. From the $\psi(2 S)$ mass value, $m_{\psi(2 S)}=3685.96 \pm 0.09 \mathrm{MeV} / c^{2}$ [12], we find $m_{X(3872)}=3873.4 \pm 1.4 \mathrm{MeV} / c^{2}$, consistent with the previous measurements by Belle [2] and CDF [3].

The measurement of the branching fraction $\mathcal{B}\left(B^{-} \rightarrow X(3872) K^{-}\right) \times \mathcal{B}\left(X(3872) \rightarrow J / \psi \pi^{+} \pi^{-}\right) \quad$ is performed with a counting technique. We select events 
in a $\pm 10 \mathrm{MeV} / c^{2}$ window around $m_{J / \psi \pi \pi}=3872 \mathrm{MeV} / c^{2}$, and find the number of events with $m_{\mathrm{ES}}>5.27 \mathrm{GeV} / c^{2}$ to be $N_{\text {data }}=63$. We estimate the number of these events due to combinatorial background $\left(N_{\text {comb }}=22.0 \pm 4.3\right)$ from a fit to the $m_{\mathrm{ES}}$ distribution (Fig. [(b)). The number of events with the same final state $B^{-} \rightarrow J / \psi K^{-} \pi^{+} \pi^{-}$, but not belonging to the $X(3872)$ signal, is estimated to be $N_{\text {peak }}=10.5 \pm 3.2$ from a fit to the $m_{\mathrm{ES}}$ distribution in the symmetric sideband $15<\left|m_{J / \psi \pi \pi}-3872\right|<45 \mathrm{MeV} / c^{2}$. The resulting number of signal events is 30.5 which agrees within the errors with the number of signal events, $25.4 \pm 8.7$, obtained from the fit to the $X(3872)$ in Figure 22 $(\mathrm{d})$. The branching fractions are determined using a frequentist confidence level 13]. This technique treats properly the small number of events and includes the systematic errors directly in the computation of confidence intervals or limits. The confidence level, $\alpha$, a function of $\mathcal{B}\left(B^{-} \rightarrow X(3872) K^{-}\right) \times \mathcal{B}\left(X(3872) \rightarrow J / \psi \pi^{+} \pi^{-}\right)$is computed as the fraction of times that a random number generated according to a Poisson distribution with a mean value of

$$
\begin{aligned}
\mu= & N_{b k g}+N_{\psi(2 S)} \epsilon_{w} \\
& \times \frac{\mathcal{B}\left(B^{-} \rightarrow X(3872) K^{-}\right) \mathcal{B}\left(X(3872) \rightarrow \mathrm{J} / \psi \pi^{+} \pi^{-}\right)}{\mathcal{B}\left(B^{-} \rightarrow \psi(2 S) K^{-}\right) \mathcal{B}\left(\psi(2 S) \rightarrow J / \psi \pi^{+} \pi^{-}\right)}
\end{aligned}
$$

exceeds the observed data. For a given value of $\mathcal{B}\left(B^{-} \rightarrow X(3872) K^{-}\right) \times \mathcal{B}\left(X(3872) \rightarrow J / \psi \pi^{+} \pi^{-}\right)$the variables $N_{b k g}, \quad N_{\psi(2 S)}, \quad \mathcal{B}\left(B^{-} \rightarrow \psi(2 S) K^{-}\right)$, and $\mathcal{B}\left(\psi(2 S) \rightarrow J / \psi \pi^{+} \pi^{-}\right)$are randomly generated to determine a value of $\mu$, which is then used in a Poisson distribution to generate a new value of the number of detected events. The generation is repeated many times and the fraction of times the random number exceeds $N_{\text {data }}=63$ yields the value of $\alpha$. The variables $N_{b k g}, \quad N_{\psi(2 S)}, \quad \mathcal{B}\left(B^{-} \rightarrow \psi(2 S) K^{-}\right)$, and $\mathcal{B}\left(\psi(2 S) \rightarrow J / \psi \pi^{+} \pi^{-}\right)$, are generated according to Gaussian distributions. The mean of $N_{\psi(2 S)}$ is 556 and $\sigma=30$. The mean of $N_{b k g}$ is $N_{\text {comb }}+N_{\text {peak }}=32.5$ and $\sigma=5.9$, which includes a systematic error on $N_{\text {peak }}$ calculated by varying the boundaries of the sideband. We use published values [12] for the remaining branching fractions and their errors, assumed to be Gaussian. Finally, $\epsilon_{w}=(92 \pm 1) \%$ is the fraction of events that fall in the $m_{J / \psi \pi \pi}$ window, from applying the same mass window cut to the $\psi(2 S)$ and assuming the same efficiency. From the values of $\mathcal{B}\left(B^{-} \rightarrow X(3872) K^{-}\right)$at which $\alpha=16 \%$ and $84 \%$ we measure

$$
\begin{aligned}
\mathcal{B}\left(B^{-} \rightarrow X(3872) K^{-}\right) \times & \mathcal{B}\left(X(3872) \rightarrow J / \psi \pi^{+} \pi^{-}\right)= \\
& (1.28 \pm 0.41) \times 10^{-5} .
\end{aligned}
$$

The probability that the observed events are a background fluctuation in the considered mass window is $5.4 \times 10^{-4}$, corresponding to 3.5 Gaussian standard deviations. As a check, we per-
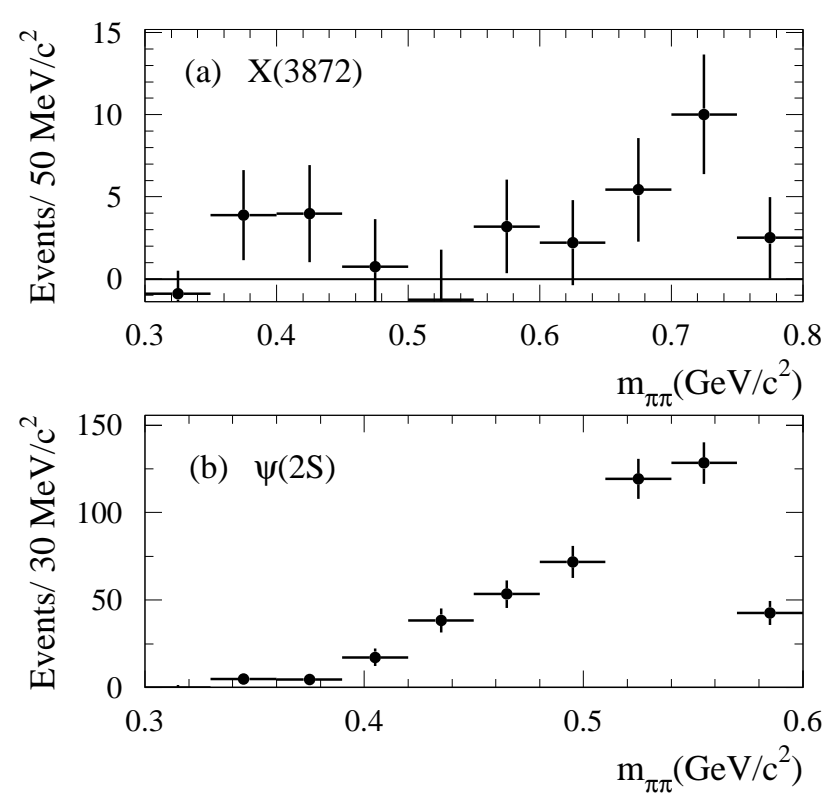

FIG. 3: Distribution of $m_{\pi^{+} \pi^{-}}$(a) at the $X(3872)$ and (b) at the $\psi(2 S)$, after subtraction of combinatorial and peaking background.

formed the same measurement on the $J / \psi \rightarrow e^{+} e^{-}$ and $J / \psi \rightarrow \mu^{+} \mu^{-}$samples separately, obtaining $\mathcal{B}\left(B^{-} \rightarrow X(3872) K^{-}\right) \times \mathcal{B}\left(X(3872) \rightarrow \mathrm{J} / \psi \pi^{+} \pi^{-}\right)=$ $(1.94 \pm 0.62) \times 10^{-5}$ and $(0.52 \pm 0.46) \times 10^{-5}$ respectively, consistent within 1.8 standard deviations.

The decay of a charmonium state into $\rho J / \psi$ is a strongly suppressed isospin-violating process. In order to investigate the nature of the $X(3872)$ state, we plot the invariant mass of the $\pi^{+} \pi^{-}$system in both the $X(3872)$ and the $\psi(2 S)$ region (Fig. 3). In the $\psi(2 S)$ case, the events are concentrated near the kinematic limit. Such behavior is not excluded for the $X(3872)$, but the statistics are too small to allow a clear conclusion. Measuring both the $m_{\pi^{+} \pi^{-}}$and angular distributions with significantly greater statistics would provide important information on the nature of the $X(3872)$.

The search for the $h_{c}$ is performed with the same frequentist technique in a $\pm 10 \mathrm{MeV} / c^{2}$ mass window centered on $m_{J / \psi \pi \pi}=3526 \mathrm{MeV} / c^{2}$ []]. With $N_{\text {data }}=9$, $N_{\text {comb }}=6.9 \pm 3.5, N_{\text {peak }}=0.6 \pm 1.5$, and assuming the same efficiency $\epsilon_{w}=(92 \pm 1) \%$, we set a $90 \%$ C.L. limit $\mathcal{B}\left(B^{-} \rightarrow h_{c} K^{-}\right) \times \mathcal{B}\left(h_{c} \rightarrow J / \psi \pi^{+} \pi^{-}\right)<3.4 \times 10^{-6}$.

The probability that we would see a signal as large as the one observed from background fluctuations alone is $39 \%$.

Finally, we search for $B^{-} \rightarrow J / \psi D^{0} \pi^{-}$decays with $D^{0} \rightarrow K^{-} \pi^{+}$. The decay $D^{0} \rightarrow K^{-} \pi^{+}$would have an r.m.s. width of $5.4 \mathrm{MeV} / c^{2}$ in $m_{K^{-} \pi^{+}}$as determined from Monte Carlo. We study this distri- 


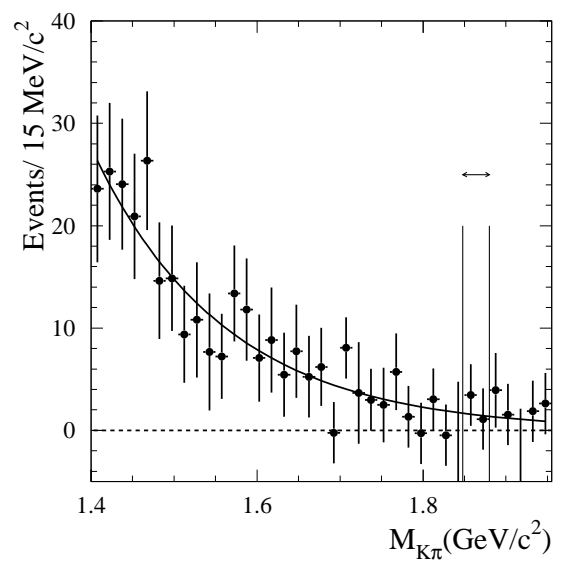

FIG. 4: Distribution of $m_{K^{-} \pi^{+}}$in events $B^{-} \rightarrow J / \psi K^{-} \pi^{+} \pi^{-}$, with combinatorial background removed. Overlaid is an exponential fit. The arrow indicates the $3 \sigma$ region expected for $D^{0} \rightarrow K^{-} \pi^{+}$.

bution in the same way we studied $m_{J / \psi \pi \pi}$. The $m_{K^{-} \pi^{+}}$combinatorial-subtracted distribution (Fig. 4) shows no significant structure, and it is therefore used to set a limit. We fit the background from other $B^{-} \rightarrow J / \psi K^{-} \pi^{+} \pi^{-}$decays with an exponential function of $m_{K^{-} \pi^{+}}$and obtain $N_{\text {peak }}=2.9 \pm 1.4$. The frequentist approach described above, with $N_{\text {data }}=10$, $N_{\text {comb }}=7.8 \pm 2.8$ and $\epsilon / \epsilon_{\psi(2 S)}=1.00 \pm 0.07$ yields the $90 \%$ C.L. limit $\mathcal{B}\left(B^{-} \rightarrow J / \psi D^{0} \pi^{-}\right)<5.2 \times 10^{-5}$. This upper limit rules out the explanation of the inclusive $J / \psi$ momentum spectrum with intrinsic charm proposed in 8].

In summary, we measured $\mathcal{B}\left(B^{-} \rightarrow J / \psi K^{-} \pi^{+} \pi^{-}\right)=$ $(116 \pm 7$ (stat. $) \pm 9$ (syst. $)) \times 10^{-5}$ with an error almost a factor two smaller than the present average [12] and we confirmed the observation of $B^{-} \rightarrow X(3872) K^{-}$[2, 3]. We measured $\mathcal{B}\left(B^{-} \rightarrow X(3872) K^{-}\right) \times \mathcal{B}(X(3872) \rightarrow$ $\left.\mathrm{J} / \psi \pi^{+} \pi^{-}\right)=(1.28 \pm 0.41) \times 10^{-5}$ and $m_{X(3872)}=$ $3873.4 \pm 1.4 \mathrm{MeV} / c^{2}$. We also studied the $m_{J / \psi \pi \pi}$ and $m_{K^{-} \pi^{+}}$distributions searching for $B^{-} \rightarrow h_{c} K^{-}$and $B^{-} \rightarrow J / \psi D^{0} \pi^{-}$decays and set limits on their branching fractions, $\mathcal{B}\left(B^{-} \rightarrow h_{c} K^{-}\right) \times \mathcal{B}\left(h_{c} \rightarrow J / \psi \pi^{+} \pi^{-}\right)<$ $3.4 \times 10^{-6}$ and $\mathcal{B}\left(B^{-} \rightarrow J / \psi D^{0} \pi^{-}\right)<5.2 \times 10^{-5}$ at $90 \%$ C.L.

We are grateful for the excellent luminosity and machine conditions provided by our PEP-II colleagues, and for the substantial dedicated effort from the computing organizations that support BABAR. The collaborating institutions wish to thank SLAC for its support and kind hospitality. This work is supported by DOE and NSF (USA), NSERC (Canada), IHEP (China), CEA and CNRS-IN2P3 (France), BMBF and DFG (Germany), INFN (Italy), FOM (The Netherlands), NFR (Norway), MIST (Russia), and PPARC (United Kingdom). Individuals have received support from CONACyT (Mexico), A. P. Sloan Foundation, Research Corporation, and Alexander von Humboldt Foundation.

* Now at Department of Physics, University of Warwick, Coventry, United Kingdom

† Also with Università della Basilicata, Potenza, Italy

‡ Also with IFIC, Instituto de Física Corpuscular, CSICUniversidad de Valencia, Valencia, Spain

$\S$ Deceased

[1] Charge-conjugate reactions are included implicitely throughout this Letter.

[2] Belle Collaboration, S. K. Choi et al., Phys. Rev. Lett. 91, 262001 (2003).

[3] CDF Collaboration, D. Acosta et al., hep-ex/0312021 submitted to Phys. Rev. Lett.

[4] E. J. Eichten, K. Lane, and C. Quigg, Phys. Rev. Lett. 89, 162002 (2002); E. J. Eichten, K. Lane, and C. Quigg, hep-ph/0401210

[5] S. Pakvasa and M. Suzuki, Phys.Lett. B 579, 67 (2004); N. A. Tornqvist, hep-ph/0308277 F. E. Close and P. R. Page, Phys.Lett. B 578, 119 (2004); E. S. Swanson, hep-ph/0311229 T. Barnes and S. Godfrey, hep-ph/0311162 M. B. Voloshin, Phys. Lett. B 579, 316 (2004).

[6] T. A. Armstrong et al., Phys. Rev. Lett. 69, 2337 (1992).

[7] BABAR Collaboration, B. Aubert et al., Phys. Rev. D 67, 032002 (2003); CLEO Collaboration, R. Balest et al., Phys. Rev. D 52, 2661 (1995).

[8] C. H. Chang and W. S. Hou, Phys. Rev. D 64, 071501 (2001).

[9] BABAR Collaboration, B. Aubert et al., Nucl. Instrum. \& Methods A 479, 1 (2002).

[10] GEANT4 Collaboration, S. Agostinelli et al., Nucl. Instrum. \& Methods A 506, 250 (2003).

[11] BABAR Collaboration, B. Aubert et al., Phys. Rev. D 65, 032001 (2002).

[12] Particle Data Group, K. Hagiwara et al., Phys. Rev. D 66, 010001 (2002), as updated for 2003 at http://pdg.lbl.gov/

[13] Particle Data Group, K. Hagiwara et al., Phys. Rev. D 66, 010001 (2002), section 31.4.2.

[14] Crystal Ball Collaboration, T. Skwarnicki, DESY F3186-02.

[15] ARGUS Collaboration, H. Albrecht et al., Z. Phys. C 48, 543 (1990). 